\title{
Caracterización de aislamientos nativos de Metarhizium spp. para el control de Mahanarva andigena en el cultivar de caña de azúcar POJ93 en la Amazonia ecuatoriana
}

\author{
Characterization of native isolates of Metarhizium spp. for the control of \\ Mahanarva andigena in the sugarcane cultivar POJ93 in the Ecuadorian Amazon
}

\author{
Segundo Valle ${ }^{1 *}$, Karina Carrera ${ }^{1}$, Reinaldo Alemán ${ }^{1}$, Willan Caicedo ${ }^{1}$
}

\begin{abstract}
RESUMEN
Este trabajo tiene como objetivo evaluar el hongo nativo Metarhizium spp. en el control del salivazo (Mahanarva andigena) de la caña de azúcar, en Pastaza, Ecuador. Para esto, se seleccionaron veinte aislamientos monospóricos obtenidos en cultivares de caña; procedentes de suelo (DAS5401, SJS5103, DAS5403, TS6302, TS6304, SJS5104, DAS5402, PS5002, TS6308, DAS5404, PS5004, SJS5102, SJS5101, PS5001, PS5003) e insectos micosados (TI6301, TI6303, TI6305, TI6306, TI6307). El experimento se realizó a través de un diseño completamente aleatorizado con seis réplicas por cada aislamiento. Los datos se procesaron por ANOVA y la comparación de medias se hizo con la prueba Tukey $(\mathrm{P}<0,05)$. El aislamiento DAS5401 presentó el mayor $(\mathrm{P}<0,0001)$ crecimiento radial $(64,7 \mathrm{~mm})$ y no difirió significativamente $(\mathrm{P}>0,05)$ de SJS5103 $(62,9 \mathrm{~mm})$, DAS5403 $(58,5 \mathrm{~mm})$ y TS6302 $(5,4 \mathrm{~mm})$. En cuanto a la tasa de crecimiento, el monospórico DAS5401 obtuvo el mayor $(\mathrm{P}<0,0001)$ crecimiento $\left(2,99 \mathrm{~mm}_{\text {día }}{ }^{-1}\right)$, sin diferencias significativas $(\mathrm{P}<0,05)$ con SJS5103 (2,90 mm.día $\left.{ }^{-1}\right)$, DAS5403 (2,68 mm.día $\left.{ }^{-1}\right)$ y TS6302 (2,62 mm.día $\left.{ }^{-1}\right)$. La mayor producción de conidios se evidenció en los monospóricos TS6302 (1,09x108 conidios.mL $\left.{ }^{-1}\right)$, SJS5101 (1,04x 108 conidios.mL $\left.{ }^{-1}\right)$, DAS5401 $\left(9,35 \times 10^{7}\right.$ conidios. $\left.\mathrm{mL}^{-1}\right)$ y PS5002 $\left(9,20 \times 10^{7}\right.$ conidios. $\left.\mathrm{mL}^{-1}\right)$. Los aislamientos monospóricos de Metarhizium spp. obtenidos del suelo y a partir de insectos micosados en cultivares de caña de azúcar en Pastaza, Ecuador, mostraron variabilidad en cuanto a crecimiento radial, tasa de crecimiento y producción de conidios, para fines de selección como micoinsecticidas de Mahanarva andigena en condiciones de laboratorio, invernadero y campo.
\end{abstract}

Palabras clave: biocontrol, caña de azúcar, conidios, entomopatógeno, monospóricos.

\section{ABSTRACT}

With the purpose of counting in Pastaza, Ecuador, with native isolates of (Metarhizium spp.) with potential to control spittlebug (Mahanarva andigena) of sugarcane, 20 monosporic isolates were obtained from sugarcane cultivars; from soil (DAS5401, SJS5103, DAS5403, TS6302, TS6304, SJS5104, DAS5402, PS5002, TS6308, DAS5404, PS5004, SJS5102, SJS5101, PS5001, PS5003) and the insects (TI6301, TI6303, TI6305, TI6306, TI6307) were characterized according to radial growth, growth rate and concentration of conidia. The experiment was conducted through a completely randomized design with six replicates for each isolation. ANOVA processed the data, the comparison of means was made with the Tukey test $(P<0.05)$. The DAS5401 isolate presented the highest $(P<0.0001)$ radial growth $(64.7 \mathrm{~mm})$ and did not differ significantly $(P>0.05)$ from SJS5103 $(62.9 \mathrm{~mm}), D A S 5403$ $(58.5 \mathrm{~mm})$ and TS6302 $(57.4 \mathrm{~mm})$. Regarding the growth rate, the monosporic DAS5401 exhibited the highest $(P<0.0001)$ growth $\left(2.99 \mathrm{~mm}\right.$ day $\left.{ }^{-1}\right)$, without significant differences $(P<0.05)$ with SJS5103 $\left(2.90 \mathrm{~mm} . \mathrm{day}^{-1}\right)$, DAS5403 $\left(2.68 \mathrm{~mm}^{\mathrm{day}}{ }^{-1}\right)$ and TS6302 $\left(2.62 \mathrm{~mm}_{\text {.day }}{ }^{-1}\right)$. The highest production of conidia was evidenced in the monosporic TS6302 (1.09x108 conidia.mL $\left.{ }^{-1}\right)$, SJS5101

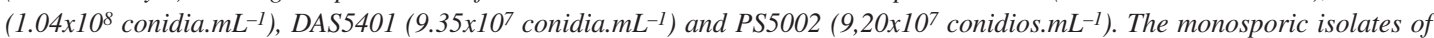
Metarhizium spp. obtained from the soil and from mycosed insects in cultivars of sugarcane in Pastaza, Ecuador, showed variability in terms of radial growth, rate of the growth and production of conidia, for selection purposes as mycoinsecticides Mahanarva andigena under laboratory, greenhouse and field conditions.

Keywords: biocontrol, sugar cane, conidia, entomopathogenic, monosporic.

\footnotetext{
1 Universidad Estatal Amazónica, Departamento de Ciencias de la Tierra, Paso Lateral S/N, Km 2²² Vía a Napo. Puyo, Pastaza, Ecuador.

* Autor por correspondencia: svalle@uea.edu.ec
}

Fecha de Recepción: 9 de octubre, 2019.

Fecha de Aceptación: 13 de septiembre, 2020. 


\section{Introducción}

La caña de azúcar (Saccharum spp. híbrido) es una poácea nativa de las regiones tropicales que se distribuye a nivel mundial. En el Ecuador, este cultivo se encuentra entre los principales rubros agrícolas y ocupa un lugar preponderante en la actividad económica y social del país por su contribución al PIB agrícola nacional (CINCAE, 2017). En la provincia de Pastaza, perteneciente a la Región Amazonía Ecuatoriana (RAE), se cultivan alrededor de 927,05 ha de caña de azúcar (POJ93). Es el principal rubro agrícola en la provincia y su producción se destina a la elaboración de panela, miel, jugos, alcohol y caña de fruta (Valle et al., 2015).

Actualmente, el cultivo de caña de azúcar en Pastaza se ha visto afectado por el salivazo (Mahanarva andigena Jacobi). Este insecto se alimenta de las láminas foliares y provoca una fitotoxemia llamada "quema de las hojas", con la constante disminución de la capacidad fotosintética de las hojas. El daño causado por el salivazo es medido con la reducción de la productividad de los tallos/planta que puede variar entre 40 y $60 \%$, y provocar la afectación en la calidad de la materia prima para el proceso agroindustrial (Mendoza et al., 2013).

Una de las estrategias básicas para el control biológico de plagas es la exploración inicial de los enemigos naturales nativos a nivel regional antes de introducir cepas exóticas (Torres et al., 2013). En la provincia de Pastaza existe gran variabilidad en cuanto a microclimas, y esto favorece la existencia de una amplia diversidad de organismos nativos con potencial de control biológico del salivazo, donde los cultivos monospóricos representan una alternativa que permite seleccionar las mejores características de los aislamientos silvestres (Cortez et al., 2003).

En estudios preliminares ejecutados por Valle et al. (2018) se demostró que es viable el uso de Metarhizium anisopliae para reducir los niveles poblacionales del salivazo en Pastaza, Sin embargo, resulta necesario disponer de una mayor cantidad de aislamientos nativos con potencialidades para el control de esta plaga.

Con estos antecedentes, en el estudio se caracterizaron veinte aislamientos nativos, monospóricos, del hongo Metarhizium anisopliae, con la finalidad de seleccionar los mejores en cuanto a características fisiológicas y producción de conidios para el manejo del salivazo (Mahanarva andigena) en cultivares de caña de azúcar en Pastaza, Ecuador.

\section{Materiales y métodos}

\section{Localización del área de estudio}

La investigación se realizó en el cantón Pastaza durante el año 2018. Esta zona corresponde a la forma de vida "Bosque siempreverde piemontano", con precipitaciones medias anuales que oscilan entre 2500 y $4000 \mathrm{~mm}$, distribuidas durante todo el año. La temperatura anual promedio en la mayor parte de la provincia de Pastaza oscila entre 24 y $28{ }^{\circ} \mathrm{C}$ y una humedad relativa cercana al $80 \%$. Los suelos en su mayoría pertenecen al orden Inceptisoles (Bravo et al., 2015).

\section{Origen de aislamientos}

Se utilizaron veinte aislamientos nativos de Metarhizium spp. procedentes del suelo y a partir de insectos micosados en cultivares de caña de azúcar, en las parroquias Tarqui, Puyo, Diez de Agosto y San José pertenecientes al cantón Pastaza, provincia de Pastaza (Tabla 1).

Tabla 1. Aislamientos monospóricos Metarhizium spp. procedentes de cuatro parroquias del cantón Pastaza, provincia de Pastaza, Ecuador.

\begin{tabular}{lll}
\hline Codificación & \multicolumn{1}{c}{ Procedencia } & \multicolumn{1}{c}{ Parroquias } \\
\hline TI6301 & Insecto Micosado & Tarqui \\
TS6302 & Suelo & Tarqui \\
TI6303 & Insecto Micosado & Tarqui \\
TS6304 & Suelo & Tarqui \\
TI6305 & Insecto Micosado & Tarqui \\
TI6306 & Insecto Micosado & Tarqui \\
TI6307 & Insecto Micosado & Tarqui \\
TS6308 & Suelo & Tarqui \\
PS5001 & Suelo & Puyo \\
PS5002 & Suelo & Puyo \\
PS5003 & Suelo & Puyo \\
PS5004 & Suelo & Puyo \\
DAS5401 & Suelo & Diez de Agosto \\
DAS5402 & Suelo & Diez de Agosto \\
DAS5403 & Suelo & Diez de Agosto \\
DAS5404 & Suelo & Diez de Agosto \\
SJS5101 & Suelo & San José \\
SJS5102 & Suelo & San José \\
SJS5103 & Suelo & San José \\
SJS5104 & Suelo & San José \\
\hline
\end{tabular}




\section{Aislamiento y obtención de cultivos monospóricos de Metarhizium spp.}

El estudio se realizó en el Laboratorio de Microbiología de la Universidad Estatal Amazónica. Para aislar hongos hifomicetos a partir de muestras de suelo se utilizó la metodología de dilución seriada (Schapovaloff et al., 2015). En las muestras de suelo se hicieron diluciones seriadas en base 10 y se utilizó la dilución $10^{-2}$, para la inoculación de $100 \mathrm{uL}$ por cada placa de Petri que contenía Potato Dextrosa Agar (PDA). A los 8 días de inoculadas las placas de Petri se aislaron e identificaron las colonias de Metarhizium spp. con base en las estructuras reproductivas (Humber, 2012). Para el aislamiento a partir de insectos micosados se utilizó la metodología de García et al. (2012a). Los salivazos infectados colectados en el campo se colocaron en un tubo eppendorf de $2 \mathrm{~mL}$ con papel filtro y se llevaron al laboratorio. Los insectos se desinfestaron superficialmente con hipoclorito de sodio al 1\%, durante $3 \mathrm{~min}$. El exceso de hipoclorito se eliminó con un lavado triple en agua destilada estéril y para evitar el exceso de humedad se colocaron los insectos sobre papel toalla estéril. Luego, cada insecto fue depositado en cámara húmeda $(90 \% \mathrm{HR})$ a $26^{\circ} \mathrm{C}$, durante ocho días. Finalmente, cuando se observó algún crecimiento de hongos sobre el cuerpo del insecto, se aisló sembrándolo en un medio de PDA y ácido láctico al 25\% adicionando el 1\% del volumen que se preparará. El aislamiento se incubó durante ocho días a $27^{\circ} \mathrm{C}$, para permitir su desarrollo hasta la esporulación e identificar el microorganismo obtenido.

Para la obtención de cultivos monospóricos de los veinte aislamientos se utilizó la metodología de Estrada et al. (1997), que consistió en lograr una concentración de $3 \times 10^{5}$ conidios. $\mathrm{mL}^{-1}$, a partir de cada uno de los aislamientos poliespóricos obtenidos, cultivados en Potato Dextrosa Agar (PDA) acidificado, mantenido a $27 \pm 1{ }^{\circ} \mathrm{C}$. La remoción de las esporas de los cultivos se realizó con Tween 80 a 1 0,05\%. A partir de esta suspensión se hicieron diluciones seriadas y con la cámara de Newbauer y un microscopio Midcis se realizó el recuento de conidios por mililitro, a fin de calcular la concentración requerida (Villamil y Martínez, 2014). De esta suspensión se tomaron $3 \mu \mathrm{L}$ y se depositaron sobre el extremo superior de una estría previamente marcada por el reverso de la caja de
Petri y con la ayuda de un asa en argolla se esparció el inóculo sobre estaestas. Por cada aislamiento se realizaron cuatro réplicas. Luego de incubar la caja de Petri a $27 \pm 1^{\circ} \mathrm{C}$ durante 24 horas, se observó la germinación de los conidios con el objetivo 10X del microscopio. Posteriormente se demarcó el área con el objetivo 40X y se cortó el bloque de agar con la ayuda de un bisturí, se cultivó en medio PDA acidificado y se incubó a una temperatura de $25^{\circ} \mathrm{C}$ con luz durante 8 días. Luego se seleccionó la colonia que tuvo un mejor crecimiento para su utilización en la evaluación de la tasa de crecimiento.

\section{Mediciones realizadas}

\section{Crecimiento radial y tasa de crecimiento}

Para determinar el crecimiento radial y la tasa de crecimiento de los aislamientos de Metarhizium spp., se procedió de la siguiente manera: con el empleo de un sacabocados se tomaron discos de $5 \mathrm{~mm}(\varnothing)$ de colonias de cuatro días de crecimiento en cada aislamiento, los discos de micelio se transfirieron usando un asa estéril y se colocaron en el centro de cada caja de Petri de $90 \mathrm{~mm}(\varnothing)$, previamente trazada en la parte externa de la base con una línea en dirección horizontal y vertical. Posteriormente se sellaron con parafilm, se rotularon y se llevaron a incubación a la incubadora Memmert por 20 días a $27 \pm 1{ }^{\circ} \mathrm{C}$ en oscuridad total. El crecimiento radial de las colonias se midió en dos direcciones perpendiculares cada 48 horas usando un calibrador Vernier digital durante 20 días. A los valores obtenidos en la medición se les restaron los $5 \mathrm{~mm}$ correspondientes a cada círculo de micelio inicial. La tasa de crecimiento se calculó al final del ensayo con la fórmula propuesta por Guigón et al. (2010).

$\begin{gathered}\text { Tasa de } \\ \text { crecimiento }\end{gathered}=\frac{\text { crecimiento final }- \text { Crecimiento inicial }}{\text { Tiempo de incubación }}$

\section{Concentración de conidios}

Para determinar la concentración de conidios por cada aislamiento se siguió la metodología de Ruiz et al. (2011). De las cajas de Petri de veinte días de incubación se procedió a obtener los conidios de la superficie del cultivo bajo condiciones asépticas, inundando la caja de Petri con $10 \mathrm{~mL}$ de 
agua destilada estéril (ADE) + Tween $80(0,1 \%)$ y raspando la colonia con un bisturí. La suspensión de conidios fue filtrada con gasa estéril para separar el micelio. Los conidios se agitaron durante 10 min en un Vórtex. Posteriormente el conteo de conidios se realizó en cámara de Neubauer improved brigh-line, utilizando la dilución $10^{-2}$, en cinco cuadrantes del cuadrado central con ayuda del objetivo 40x del microscopio. La concentración de conidios por $\mathrm{mL}^{-1}$ se estimó mediante la fórmula:

$$
\begin{gathered}
\text { Concentración } \\
\text { de conidios } / \mathrm{mL}
\end{gathered}=\frac{\text { Total de células contadas } \mathrm{x} 250.000}{\text { Número de cuadros } \mathrm{x} \text { Dilución }}
$$

\section{Análisis estadístico}

Los datos de crecimiento radial, tasa de crecimiento y producción de conidios fueron sometidos a análisis de varianza de una vía (ANOVA). En los casos en que se encontraron diferencias significativas $(P<0.05)$, las medias se compararon por la prueba de Tukey. Todos los análisis se realizaron con el sistema estadístico SPSS versión 22.

\section{Resultados y discusión}

En la Figura 1 se observa el crecimiento radial del micelio de aislamientos de Metarhizium spp. a los 20 días de desarrollo en medio de cultivo PDA a $27 \pm 1^{\circ} \mathrm{C}$. El aislamiento DAS5401 presentó el mayor $(\mathrm{P}<0,0001)$ crecimiento radial $(64.7 \mathrm{~mm})$ y no difirió significativamente $(\mathrm{P}>0,05)$ de los aislamientos SJS5103 (62.9 mm), DAS5403
$(58,5 \mathrm{~mm})$ y TS6302 $(57.4 \mathrm{~mm})$, los cuales superaron a los aislamientos TI6301 (47,6 mm), SJS5104 (46 mm), TI6307 (46 mm), DAS5402 (45.1 mm), PS5002 (45 mm), TI6306 (44 mm), TS6308 (43,5 mm), DAS5404 (43,4 mm), TI6305 (41,7 mm), PS5004 (39,2 mm), SJS5102 (35,8 mm), TI6303 (33,8 mm), SJS5101 (33,7 mm), PS5001 $(33,5 \mathrm{~mm})$, TS6304 (31,1 mm) y PS5003 $(30,5 \mathrm{~mm})$ respectivamente.

Los aislamientos monospóricos: DAS5401, SJS5103, DAS5403, TS6302 presentaron el mayor $(\mathrm{P}<0,0001)$ crecimiento radial del micelio. El desarrollo del micelio es una característica importante para la selección y producción de hongos entomopatógenos (Ekesi et al., 1999), y su respuesta a la temperatura está descrita por la zona de origen del aislamiento y por aspectos genéticos (Dimbi et al., 2004). La respuesta ante las fluctuaciones de temperatura se considera como un punto de partida para la selección de cepas fúngicas con capacidad para el control biológico, ya que esta incide en el crecimiento vegetativo y persistencia en el campo, y por consiguiente en su eficacia en campo (Souza et al., 2014).

Las temperaturas adecuadas para el crecimiento de $M$. anisopliae fluctúan entre 25 y $30^{\circ} \mathrm{C}$, mientras que las temperaturas extremas $\left(<15^{\circ}\right.$ y $>32^{\circ} \mathrm{C}$ ) pueden retrasar su desarrollo o capacidad para esporular. Al respecto, existen estudios que comprueban que hay cepas que varían en su tolerancia a un mayor rango de temperatura. Es por ello que Nussenbaum et al. (2013) clasificaron cepas de $M$. anisopliae según la temperatura óptima para el crecimiento como frío activo (capaz de

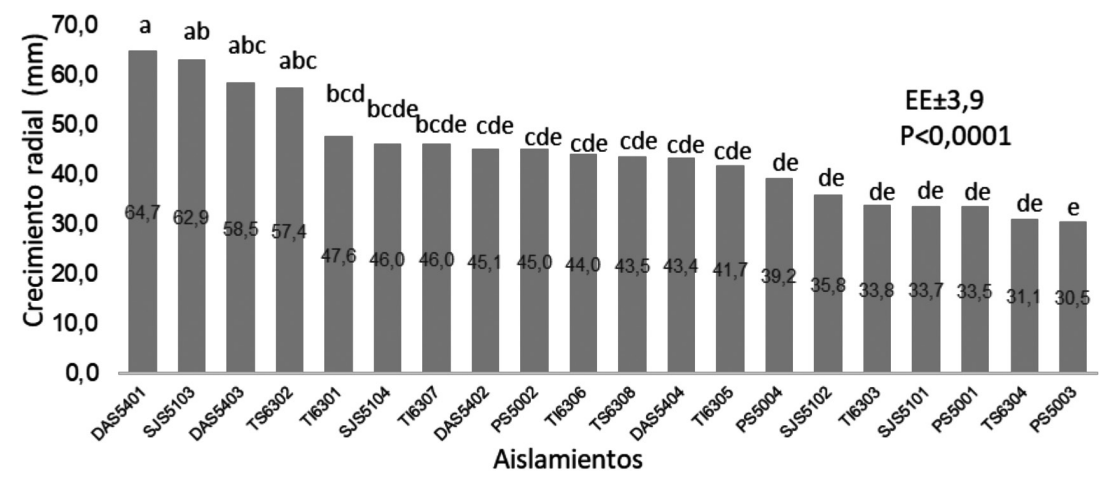

Figura 1. Crecimiento radial de aislamientos de Metarhizium spp. a los 20 días de desarrollo en medio de cultivo PDA a $27 \pm 1^{\circ} \mathrm{C}$.

Medias con letras distintas indican diferencias significativas para el test de Tukey $(p<0,05)(n=6)$. 
germinar a $5^{\circ} \mathrm{C}$ ), calor activo (capaz de germinar a $37^{\circ} \mathrm{C}$ ) y meso-termo activo (incapaz de germinar a cualquiera de estas temperaturas).

Por otra parte, cuando el hongo se desarrolla en el campo, la humedad y la radiación UV también se predisponen como factores abióticos importantes, que limitan el uso efectivo de hongos entomopatógenos, pues estos interactúan para incidir sobre la germinación de los conidios en condiciones naturales (Chandra y Rahman, 2016).

En la Figura 2 se muestra la tasa de crecimiento de aislamientos de Metarhizium spp. a los 20 días de desarrollo en medio de cultivo PDA a $27 \pm 1{ }^{\circ} \mathrm{C}$. El aislamiento DAS5401 presentó la mayor $(\mathrm{P}<0,0001)$ tasa de crecimiento $(2,99 \mathrm{~mm}$. día $\left.{ }^{-1}\right)$, y no difirió significativamente $(\mathrm{P}<0,05)$ de los aislamientos SJS5103 (2,90 mm.día ${ }^{-1}$ ), DAS5403 (2,68 mm.día $\left.{ }^{-1}\right)$ y TS6302 $(2,62 \mathrm{~mm}$. día $\left.^{-1}\right)$, y fueron superiores a los aislamientos TI6301 (2,13 mm día $\left.{ }^{-1}\right)$, SJS5104 (2,05 mm.día $\left.{ }^{-1}\right)$, TI6307 (2,05 mm.día $\left.{ }^{-1}\right)$, DAS5402 (2,01 mm.día $\left.{ }^{-1}\right)$, PS5002 (2 mm.día $\left.{ }^{-1}\right)$, TI6306 (1.95 mm.día $\left.{ }^{-1}\right)$, TS6308 (1,93 mm.día $\left.{ }^{-1}\right)$, DAS5404 (1.92 mm.día $\left.{ }^{-1}\right)$, TI6305 (1,84 mm.día $\left.{ }^{-1}\right)$, PS5004 (1,71 mm.día $\left.{ }^{-1}\right)$, SJS5102 (1,54 mm.día $\left.{ }^{-1}\right)$, TI6303 (1,44 mm.día $\left.{ }^{-1}\right)$, SJS5101 (1,44 mm.día $\left.{ }^{-1}\right)$, PS5001 (1,43 mm.día $\left.{ }^{-1}\right)$, TS6304 (1,31 mm.día $\left.{ }^{-1}\right)$, PS5003 (1,28 mm.día $\left.{ }^{-1}\right)$.

Los aislamientos monospóricos: DAS5401, SJS5103, DAS5403 y TS6302 presentaron la mayor tasa de crecimiento, superior a lo determinado por García et al. (2012b), quienes encontraron tasas de crecimiento de 1,5 y 1,8 mm.día ${ }^{-1}$, al evaluar diferentes aislamientos de M. anisopliae.
En un estudio desarrollado por Ummidi et al. (2013) con Metarhizium y Beauveria se demostró que las cepas más virulentas presentan un crecimiento rápido en comparación con las menos virulentas. Aunque el crecimiento de los hongos puede no tener influencia directa en su virulencia (Mwamburi et al., 2015) señalan que las altas concentraciones de surfactantes de los medios de cultivo y elevadas densidades de esporas provocan un crecimiento reducido.

En la Figura 3 se observa la producción de conidios de aislamientos de Metarhizium spp. a los 20 días de desarrollo en medio de cultivo PDA a $27 \pm 1{ }^{\circ} \mathrm{C}$. No hubo diferencias significativas $(\mathrm{P}>0,05)$ entre los aislamientos TS6302 $\left(1,09 \times 10^{8}\right.$ conidios.mL-1), SJS5101 (1,04 x $10^{8}$ conidios. $\left.\mathrm{mL}^{-1}\right)$, DAS5401 $\left(9,35 \times 10^{7}\right.$ conidios. $\left.\mathrm{mL}^{-1}\right)$, PS5002 (9,20 x $10^{7}$ conidios. $\left.\mathrm{mL}^{-1}\right)$, los cuales superaron a los aislamientos TI6301 $\left(6,45 \times 10^{7}\right.$ conidios $\left.\mathrm{mL}^{-1}\right)$, SJS5103 $\left(3,74 \times 10^{7}\right.$ conidios. $\left.\mathrm{mL}^{-1}\right)$, SJS5102 (3,06 x $10^{7}$ conidios.mL $\left.{ }^{-1}\right)$, PS5003 $\left(3,06 \times 10^{7}\right.$ conidios.mL $\left.{ }^{-1}\right)$, TS6304 $\left(2,95 \times 10^{7}\right.$ conidios. $\left.\mathrm{mL}^{-1}\right)$, SJS5104 (2,66 x $10^{7}$ conidios. $\left.\mathrm{mL}^{-1}\right)$, DAS5404 (1,85 x $10^{7}$ conidios. $\left.\mathrm{mL}^{-1}\right)$, DAS5402 (1,61 x $10^{7}$ conidios.mL $\left.{ }^{-1}\right)$, PS5004 $\left(1,17 \times 10^{7}\right.$ conidios.mL $\left.{ }^{-1}\right)$, PS5001 $\left(1,11 \times 10^{7}\right.$ conidios. $\left.\mathrm{mL}^{-1}\right)$, DAS5403 $\left(1,02 \times 10^{7}\right.$ conidios. $\left.\mathrm{mL}^{-1}\right)$, TI6303 (9,50 x $10^{6}$ conidios.mL $\left.\mathrm{mL}^{-1}\right), \mathrm{TI} 6305$ $\left(6,10 \times 10^{6}\right.$ conidios.mL $\left.{ }^{-1}\right)$, TI6306 $\left(5,60 \times 10^{6}\right.$ conidios. $\left.\mathrm{mL}^{-1}\right)$, TI6307 (2,90 x $10^{6}$ conidios. $\left.\mathrm{mL}^{-1}\right)$, TS6308 (2,40 x $10^{6}$ conidios. $\left.\mathrm{mL}^{-1}\right)$.

Los aislamientos nativos de Metarhizium spp. TS6302, SJS5101, DAS5401 y PS5002 presentaron

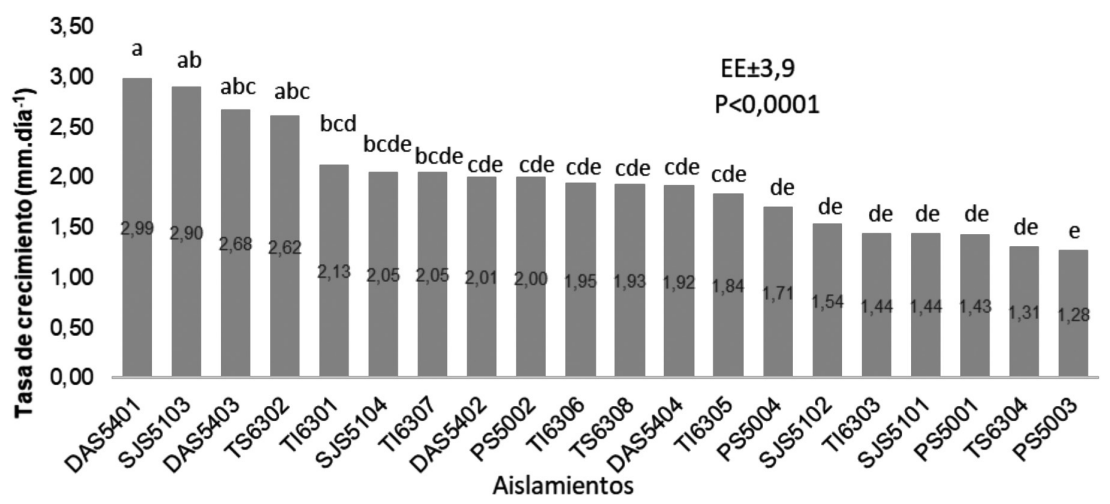

Figura 2. Tasa de crecimiento de los aislamientos de Metarhizium spp. sobre PDA a los 20 días de desarrollo a $27 \pm 1^{\circ}$ Medias con letras distintas indican diferencias significativas para el test de Tukey $(p<0,05)(n=6)$. 


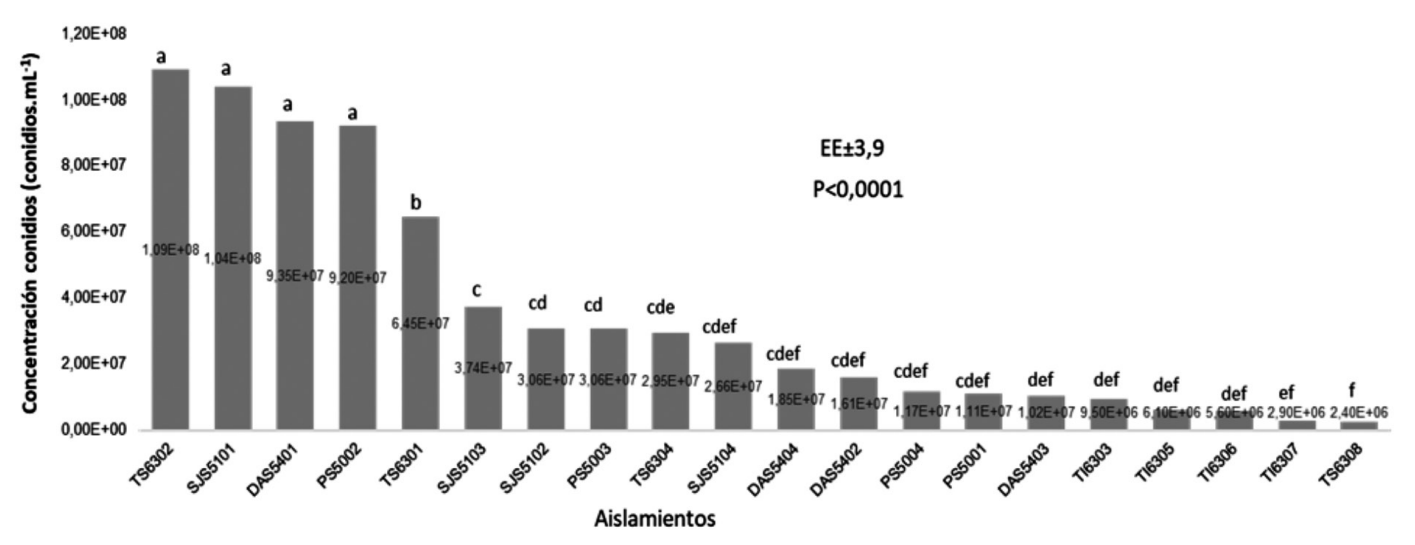

Figura 3. Concentración de conidios por cada aislamiento de Metarhizium spp. sobre medio de cultivo PDA a los 20 días de desarrollo a $27 \pm 1{ }^{\circ} \mathrm{C}$. Medias con letras distintas indican diferencias significativas para el test de Tukey $(p \leq 0,05)(n=6)$.

la mayor concentración de conidios a los 20 días de desarrollo. La selección de aislamientos con buen potencial en cuanto a producción de conidios, es un aspecto básico para la producción a escala comercial de hongos entomopatógenos (Castro et al., 2013). En este sentido, García et al. (2012a) señalan que una alta producción de conidios hace más viable su comercialización y reduce los costos de implementación en el campo.

La selección de una cepa potente a partir de formas de población fúngica es la base para su optimización genética y prospectiva de uso de gran interés como posibles criterios para la mejora de micoinsecticidas (Oliveira et al., 2015). Rojas et al. (2017) destacan que la reproducción de $M$. anisopliae no requiere de alta tecnología, por lo que constituye una alternativa viable para su uso en la agricultura.

\section{Conclusión}

Los aislamientos monospóricos de Metarhizium spp. obtenidos del suelo y a partir de insectos micosados en cultivares de caña de azúcar en Pastaza, Ecuador, mostraron variabilidad en cuanto a crecimiento radial, tasa de crecimiento y producción de conidios, para fines de selección como micoinsecticidas de Mahanarva andigena en condiciones de laboratorio, invernadero y campo.

\section{Agradecimientos}

Los autores de este trabajo desean expresar un profundo agradecimiento a la Secretaría Nacional de Educación Superior, Ciencia y Tecnología (SENESCYT) de Ecuador por el financiamiento de esta investigación.

\section{Literatura Citada}

Bravo, C.; Benítez, D.; Vargas, J.; Alemán, R.; Torres, B.; Marín, H.

2015. Caracterización socio-ambiental de unidades de producción agropecuaria en la Región Amazónica Ecuatoriana: Caso Pastaza y Napo. Revista Amazónica Ciencia y Tecnología, 4(1): 3-31.

Castro, S.M.; González, R.; Castaño, J.; Sanjuán, T.

2013. Evaluación de medios de cultivo para inducir esporulación de Isaria Tenuipes Peck. Agron., 21(1): 19-25.

Chandra, K.N.P.; Rahman, S.J.

2016. Characterisation and evaluation of Metarhizium anisopliae (Metsch.) Sorokin strains for their temperature tolerance. Mycology, 7(4): 171-179.
CINCAE (Centro de Investigación de la Caña de Azúcar del Ecuador).

2017. Informe Anual 2016. El Triunfo, Ecuador. 70 p. ISSN 13903365

Cortez, M.H.; Ortiz, G.C.F.; Alatorre, R.R.; Bravo, M.H.; Mor

A,A.G.; Aceves, N.L.A.

2003. Caracterización cultural de cepas de Lecanicillium (Verticillium) lecanii (Zimm.) Zare y Gams y su patogenicidad sobre Toxoptera aurantii Boyer. Revista Mexicana de Fitopatología, 21(2): 161-167.

Dimbi, S.; Maniania, N.K.; Lux, S.A.; Mueke, J.M. 2004. Effect of constant temperatures on germination, radial growth and virulence of Metarhizium anisopliae to 
three species of African tephritid fruit flies. Biocontrol, 49: 83-94.

Ekesi, S.; Maniania, N.K.; Ampo, N.K.

1999. Effect of temperature on germination, radial growth and virulence of Metarhizium anisopliae and Beauveria bassiana on Megalurothrips sjostedti. Biocontrol Science and Technology, 9(2): 177-185.

Estrada, M.N.; Vélez, P.E.; Montoya, E.C.

1997. Caracterización de cultivos monoespóricos del hongo Beauveria bassiana. Cenicafé, 048(04): 217-224.

García, M.; Cappello, S.; Lesher, J.; Molina, R.

2012a. Aislamiento y caracterización morfológica de los hongos entomopatógenos Beauveria bassiana y Metarhizium anisopliae. Horizonte Sanitario, 10(2): 21-28.

García, A.M.; Bustillo, A.E.; Castro, U.; Arenas, Y.

2012b. Selección de hongos entomopatógenos para controlar salivazos (Hemiptera: Cercopidae) de la caña de azúcar en Colombia. Revista Colombiana de Entomología., 38(2): 252-259.

Guigón-López, C.; Guerrero-Prieto, V.; Vargas-Albores, F.; Carvajal-Millán, E.; Ávila-Quezada, G.; Bravo-Luna, L.; Ruocco, M.; Lanzuise, S.; Woo, S.; Lorito, M.

2010. Identificación molecular de cepas nativas de Trichoderma spp. su tasa de crecimiento in vitro y antagonismo contra hongos fitopatógenos. Revista Mexicana de Fitopatología, 28(2): 87-96.

Humber, R.A.

2012. Identification of entomopathogenic fungi. En: Lacey, L.A. (Ed.). Manual of Techniques in Invertebrate Pathology (Second Edition) San Diego: Academic Press. Pp. 151-187. Mendoza, J.; Gualle, D.; Gómez, P.

2013. Plagas potenciales: una amenaza para el cultivo de la caña de azúcar en Ecuador. En Memorias del III Congreso de la Caña de Azúcar, sus Derivados y Bioenergía, realizado por la Asociación Ecuatoriana de Tecnólogos Azucareros, Guayaquil, Ecuador.

Mwamburi, L.A.; Laing, M.D.; Miller, R.M.

2015. Effect of surfactants and temperature on germination and vegetative growth of Beauveria bassiana. Brazilian Journal of Microbiology, 46(1): 67-74.

Nussenbaum, A.L.; Lewylle, M.A.; Lecuona, R.E.

2013. Germination, radial growth and virulence to boll weevil of entomopathogenic fungi at different temperatures. World Appl Sci J., 25: 1134-1140.

Oliveira, D.G.; Pauli, G.; Mascarin, G.M.; Delalibera, I. 2015. A protocol for determination of conidial viability of the fungal entomopathogens Beauveria bassiana and
Metarhizium anisopliae from commercial products. Journal of Microbiological Methods, 119: 44-52.

Rojas-Gutiérrez, R.L.; Loza-Murguia, M.; Vino-Nina, L.; Serrano-Canaviri, T.

2017. Capacidad biocontroladora de Beauveria brongniartii (Sacc.) y Metarhizium anisopliae (Metsch.,) en el control de pulgones Macrosiphum euphorbiae (Hemiptera: Aphididae). Journal of the Selva Andina Research Society, 8(1): 48-68.

Ruiz-Sánchez, E.; Chan-Cupul, W.; Pérez Gutiérrez, A.; CristóbalAlejo, J.; Uch-Vázquez, B.; Tun-Suárez, J.; Munguía-Rosales, R. 2011. Crecimiento, esporulación y germinación in vitro de cinco cepas de Metarhizium y su virulencia en huevos y ninfas de Bemisia tabaci. Revista mexicana de micología, 33: 9-15.

Schapovaloff, M.E.; Angeli, L.F.; Urrutia, M.; López, C.

2015. Ocurrencia natural de hongos entomopatógenos en suelos cultivados con yerba mate (Ilex paraguariensis St. Hil.) en Misiones, Argentina. Revista Argentina de Microbiología., 47(2): 138-142.

Souza, R.; Azevedo, R.; Lobo, A.; Rangel, D.

2014. Conidial water afinity is an important characteristic for termotolerance in entomopathogenic fungi. Biocontrol Science and Technology, 24(4): 448-461.

Torres, M.; Cortez, H.M.; Ortiz, C.F.; Capello, S.; De la Cruz, A. 2013. Caracterización de aislamientos nativos de Metarhizium anisopliae y su patogenicidad hacia Aeneolamia postica, en Tabasco, México. Revista Colombiana de Entomología, 39(1): 40-46.

Ummidi, V.R.S.; Josyula, U.; Vadlamani, P.

2013. Germination rates of Beauveria bassiana and Metarhizium anisopliae its possible correlation with virulence against Spodoptera litura larvae. Int J Adv Res., 2: 625-630.

Valle, S.; Iparraguirre, M.; Puertas, A.; Rodríguez, S.; Fiallos, A.; Hidalgo, L.; Miranda, I.

2015. Evaluación de dos métodos de monitoreo de Mahanarva andigena Jacobi en el cultivo de la caña de azúcar (Saccharum spp., híbrido) en la provincia de Pastaza, Ecuador. Rev. Protección Veg., 30(3): 185-192.

Valle-Ramírez, S.; Puertas-Arias, A.; Iparraguirre-Cruz, M.A.; Caicedo-Quinche, W.; Uvidia-Cabadiana, H.; Carrera-Sánchez, K. 2018. Nymph control of Mahanarva andigena with Metarhizium anisopliae in greenhouse and field conditions in Pastaza, ECUADOR. Tropical and Subtropical Agroecosystems, 21: 25-29.

Villamil, J.E.; Martínez, J.W.

2014. Evaluación de aislamientos nativos de Beauveria spp. sobre Tecia solanivora (Lepidoptera: Gelechiidae) in vitro. Revista de ciencias agrícolas, 31(1): 92-105. 
